\title{
Article \\ Effect of Surrounding Solvents on Interfacial Behavior of Gallium-Based Liquid Metal Droplets
}

\author{
Ji-Hye Kim ${ }^{1,+}$, Ye-Jin Park ${ }^{2,+}$, Sooyoung Kim ${ }^{3}$, Ju-Hee So ${ }^{4, *}$ and Hyung-Jun Koo ${ }^{2, *}$ (ID \\ 1 Department of New Energy Engineering, Seoul National University of Science and Technology, \\ 232 Gongneung-ro, Nowon-gu, Seoul 01811, Korea; gh5289@naver.com \\ 2 Department of Chemical and Biomolecular Engineering, Seoul National University of Science and Technology, \\ 232 Gongneung-ro, Nowon-gu, Seoul 01811, Korea; pkpk22294@gmail.com \\ 3 Department of Chemical and Biomolecular Engineering, North Carolina State University, \\ Raleigh, NC 27695, USA; skim94@ncsu.edu \\ 4 Material and Component Convergence R\&D Department, Korea Institute of Industrial Technology, \\ Ansan 15588, Korea \\ * Correspondence: jso@kitech.re.kr (J.-H.S.); hjkoo@seoultech.ac.kr (H.-J.K.) \\ + These authors contributed equally to this work.
}

Citation: Kim, J.-H.; Park, Y.-J.; Kim, S.; So, J.-H.; Koo, H.-J. Effect of Surrounding Solvents on Interfacial Behavior of Gallium-Based Liquid Metal Droplets. Materials 2022, 15, 706. https://doi.org/10.3390/ ma15030706

Academic Editor: Martin H.G. Prechtl

Received: 30 December 2021

Accepted: 14 January 2022

Published: 18 January 2022

Publisher's Note: MDPI stays neutral with regard to jurisdictional claims in published maps and institutional affiliations.

Copyright: (C) 2022 by the authors. Licensee MDPI, Basel, Switzerland. This article is an open access article distributed under the terms and conditions of the Creative Commons Attribution (CC BY) license (https:// creativecommons.org/licenses/by/ $4.0 /)$.

\begin{abstract}
Gallium-based liquid metal (GaLM) alloys have been extensively used in applications ranging from electronics to drug delivery systems. To broaden the understanding and applications of GaLMs, this paper discusses the interfacial behavior of eutectic gallium-indium liquid metal (EGaIn) droplets in various solvents. No significant difference in contact angles of EGaIn is observed regardless of the solvent types. However, the presence or absence of a conical tip on EGaIn droplets after dispensing could indirectly support that the interfacial energy of EGaIn is relatively low in non-polar solvents. Furthermore, in the impact experiments, the EGaIn droplet bounces off in the polar solvents of water and dimethyl sulfoxide (DMSO), whereas it spreads and adheres to the substrate in the non-polar solvents of hexane and benzene. Based on the dimensionless We number, it can be stated that the different impact behavior depending on the solvent types is closely related to the interfacial energy of EGaIn in each solvent. Finally, the contact angles and shapes of EGaIn droplets in aqueous buffer solutions with different $\mathrm{pH}$ values $(4,7$, and 10$)$ are compared. In the $\mathrm{pH}$ 10 buffer solution, the EGaIn droplet forms a spherical shape without the conical tip, representing the high surface energy. This is associated with the dissolution of the "interfacial energy-reducing" surface layer on EGaIn, which is supported by the enhanced concentration of gallium ion released from EGaIn in the buffer solution.
\end{abstract}

Keywords: liquid metal; interfacial behavior; solvents; impact dynamics; contact angles

\section{Introduction}

Gallium-based liquid metal (GaLM) alloys in a liquid state at room temperature have attracted considerable attention because of their fluidity, low viscosity, low toxicity, high electrical/thermal conductivity, and deformability. When exposed to oxygen, a gallium oxide layer with a thickness of 1-3 nm forms on the GaLM surface, lowering the surface tension to $\sim 0$ [1-4]. Because of the low surface tension, the metals can be patterned into various structures, allowing GaLM to be used in flexible and stretchable electronics, strain or pressure sensors, and biomedical applications [2,5-10]. The metals have been handled and used in an inert gas environment for applications that require the oxide-free, metallic surface of GaLMs [11,12].

The surface of GaLMs is significantly affected by the type and composition of the liquid environment as well as the gas environment. For example, a gelatinous gallium oxide hydroxide $(\mathrm{GaOOH})$ with lower mechanical strength than gallium oxide is formed in deionized (DI) water $[13,14]$. The gallium oxide skin is reduced in a strong acid or strong 
base, exposing the pure GaLM to the solvent $[15,16]$. In addition to aqueous solvents, the surface of GaLMs can also be manipulated when exposed to organic solvents. Lin et al., for example, reported a carbon layer-coated GaLM in ethanol [17]. Furthermore, organic solvents are also commonly used as dispersion media of GaLM micro-/nano-particles for applications of printable inks and electrolytes of energy devices [18-20]. To improve the understanding and applications of GaLMs, it is important to know the interfacial behavior of the metals in various solvents. However, to date, few studies on this topic have been conducted.

Herein, we present the interfacial behavior of a eutectic gallium-indium alloy (EGaIn, $75.5 \% \mathrm{Ga}$ and $24.5 \%$ In by weight) on a Si wafer in various solvents, including polar (DI water, ethanol, dimethyl sulfoxide (DMSO)), nonpolar (hexane, benzene, silicone oil), and $\mathrm{pH}$ buffer solutions ( $\mathrm{pH} 4,7,10)$. EGaIn is one of the most widely used GaLMs because, at room temperature, it is a homogeneous liquid at the eutectic composition. To reduce complexity, this study focuses on the interfacial characteristics of EGaIn, which is the gallium-indium binary alloy, as a model GaLM rather than gallium-indium-tin ternary alloy (i.e., Galinstan). To investigate the interfacial properties of EGaIn, we measured contact angles and conducted an impact experiment with EGaIn droplets in the various solvents. It was examined whether the presence or absence of the conical tip of EGaIn extruded from the syringe needle is determined by types of solvents with different dipole moments. Furthermore, the different impact behaviors of the EGaIn droplets were observed when they drop on the substrate in the solvents. Finally, the contact angles of the EGaIn

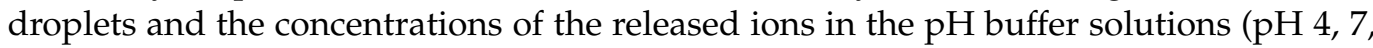
and 10) were measured to analyze how the acidic, neutral, and basic aqueous solvent environments affect the surface of the metal droplets, as well as their interfacial properties.

\section{Materials and Methods}

\subsection{Materials}

EGaIn (Gallium 75.5\%, Indium 24.5\%, >99.99\%) and silicone oil (for oil bath, $100 \mathrm{mPa} \cdot \mathrm{s}$, product no. 85409) were purchased from Sigma Aldrich (St. Louis, MO, USA). DMSO $(>99.5 \%)$, benzene $(>99.5 \%)$, and n-hexane $(>95 \%)$ were purchased from Daejung Chemicals \& Metals (Siheung, Korea). Ethanol ( $>95.0 \%)$, and $\mathrm{pH} \mathrm{4,} \mathrm{7,} \mathrm{and} 10$ buffer solutions were obtained from Samchun Chemicals (Seoul, Korea). DI water $(\geq 18 \mathrm{M} \Omega \mathrm{cm})$ was produced using a Millipore/Direct-Q3UV Water purification system (Burlington, MA, USA).

\subsection{Measurement of Contact Angles and Impact Dynamics}

The contact angles of EGaIn were measured using a drop shape analyzer (DSA100S, KRUSS, Germany) at $20{ }^{\circ} \mathrm{C}$ and analyzed using ImageJ (Java-based image processing program). A Si wafer was placed in a $5 \times 5 \times 5 \mathrm{~cm}^{3}$ cubic glass chamber and various solvents were poured into it. Subsequently, $4-5 \mu \mathrm{L}$ of the EGaIn droplet was placed on the Si wafer through a $25 \mathrm{G}$ syringe needle (outer diameter: $0.5 \mathrm{~mm}$; inner diameter: $0.26 \mathrm{~mm}$ ) in each solvent. For the impact dynamics analysis, EGaIn was dropped from a $22 \mathrm{G}$ syringe needle (outer diameter: $0.72 \mathrm{~mm}$; inner diameter: $0.41 \mathrm{~mm}$ ) at $1 \mathrm{~cm}$ from the $S i$ wafer immersed in each solvent at $20^{\circ} \mathrm{C}$. The impact behavior of the droplets was recorded using a Canon digital camera (Digital SLR Camera EOS 700D).

\subsection{Elemental Analysis}

Initially, $50 \mu \mathrm{L}$ of the EGaIn droplet was placed in a vial filled with $3 \mathrm{~mL}$ of each $\mathrm{pH}$ buffer solution. The samples of the buffer solutions with the eluted ions were taken at given time points up to $24 \mathrm{~h}$ at $20^{\circ} \mathrm{C}$. The ion concentrations of the samples were measured using an inductively coupled plasma-mass spectrometer (iCAP-Q, Thermo Fisher Scientific, Waltham, WA, USA). 


\section{Results and Discussion}

As one of the most direct and intuitive approaches to identifying the interfacial energy, the contact angles of the EGaIn droplets were measured in different types of polar and non-polar solvents. In the case of the polar solvents, we selected water, DMSO, and ethanol. The values for their relative polarity are 1.000, 0.444 , and 0.654 , respectively [21]. We selected benzene, hexane, and silicone oil as non-polar solvents. Benzene and hexane have relative polarities of 0.111 and 0.009 , respectively, and silicone oil has a low polarity [21-23]. Based on Young's equation, the contact angle on an ideal flat solid surface is determined by the interfacial tensions of three immiscible phases [24]. Therefore, we hypothesized that the contact angles of the EGaIn droplets would vary depending on the polarity of solvents due to the different interfacial energy values between the solvents and EGaIn. Figure 1a shows the contact angles of the EGaIn droplets in various polar and non-polar solvents. Contrary to our expectation, all EGaIn droplets show high contact angles at $\sim 160{ }^{\circ} \mathrm{C}$ with negligible difference regardless of the solvent types. This result may be associated with the viscoelastic thin layer newly formed on the EGaIn droplet in solvents. For EGaIn droplets in solvents, the surface of the metal could be coated with organic or inorganic layers such as oxides, hydroxides, self-assembled monolayers, and their composites, depending on the types of surrounding solvents [25-27]. It is known that gallium-rich surface layers formed on EGaIn readily adhere to a wide range of surfaces, including metals, metal oxides, plastics, and glass, as well as the native silicon oxide on the Si wafer. Consequently, when the EGaIn droplet is placed on the Si wafer, the surface layer adhering to the substrate (1) pins the EGaIn droplet to the contact line and (2) withstands the tensile stress to yield the surface layer (Figure 2) [28]. For this case, the contact angle behaves similarly to an advancing angle rather than a static contact angle. Thus, because of the sticky, solid-state surface layer formed on the EGaIn droplet in the solvents, it would be difficult to determine the interfacial energy of the EGaIn/solvents interface by the contact angle measurement.

(a)

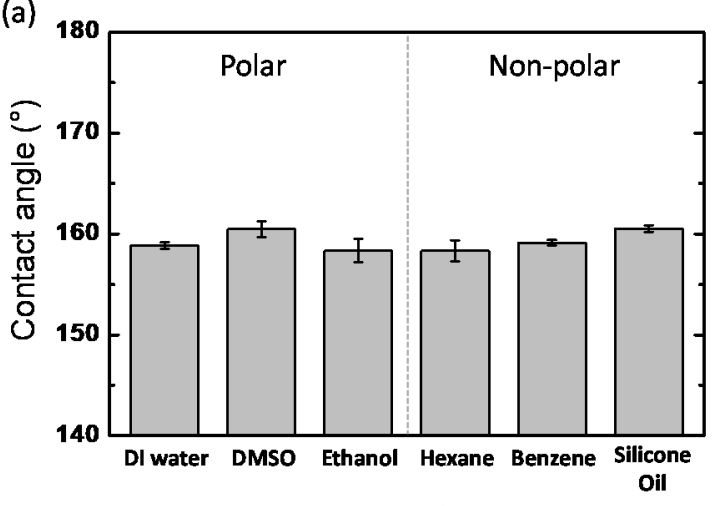

(b) DI water

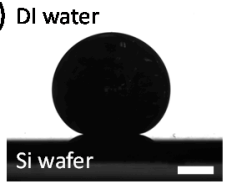

DMSO

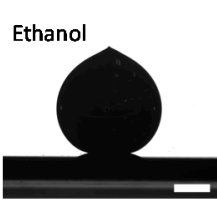

(c)

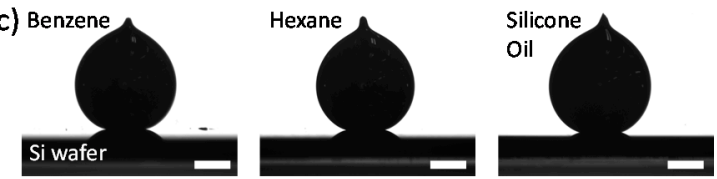

Figure 1. (a) Contact angles of EGaIn droplets on a Si wafer in polar and non-polar solvents. (b,c) Sideview images of EGaIn droplets placed on a Si wafer in (b) polar and (c) non-polar solvents. The scale bars are $0.5 \mathrm{~mm}$. The contact angles were measured at $20^{\circ} \mathrm{C}$. 


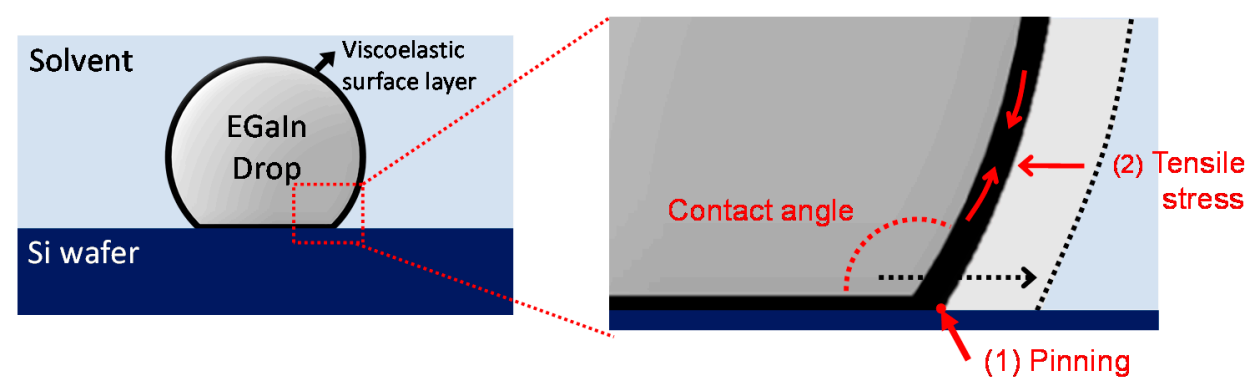

Figure 2. Schematic of the contact angle of the EGaIn droplet placed on a Si wafer. The viscoelastic surface layer (1) pins the metal to the substrate and (2) withstands the tensile stress.

Notably, the side-view images of the EGaIn droplets in the non-polar solvents differ from those in the polar solvents. In the polar solvents, except in ethanol, the EGaIn droplets have a spherical shape (Figure 1b), whereas in the non-polar solvents, the EGaIn droplets have conical tips on the top (Figure 1c). The conical tip forms when EGaIn bifurcates and is separated at the end of the needle by Plateau-Rayleigh instability [29] as the syringe needle is retracted. The presence or absence of the conical tip allows us to indirectly estimate the interfacial energy. The conical tip of the EGaIn droplet in the non-polar solvents in Figure $1 \mathrm{c}$ implies that the surface of the droplets has been modified to reduce the interfacial energy; otherwise, the droplets would form spherical shapes because of the strong cohesive force between the metal elements of EGaIn. The droplet in ethanol also has a little conical tip on the top presumably due to low interfacial energy compared to those in other polar solvents. Because the surface $\mathrm{Ga}$ atoms of EGaIn readily form $\mathrm{Ga}-\mathrm{O}$ bonds [27,30], the polar solvents containing oxygen atoms may cover the droplet's surface and cause it to behave similarly to a micelle. In contrast, in the non-polar solvents such as hexane and benzene, the surface of the EGaIn droplets is not likely to react with the solvent molecules to form Ga-C bonds [31]; however, it is still possible that the solvent molecules physically adsorb to the surface of the droplet. Thus, the interfacial energy of the EGaIn droplets in solvents is influenced by the types and polarity of solvents.

Analyzing the impact dynamics of droplets could be another way to examine the interfacial energy. The droplet impact dynamics, such as splash, bouncing, spreading, and adhesion, strongly depend on interfacial tensions as well as the impact velocity $[32,33]$. We observed the impact behavior of an EGaIn droplet falling on a Si wafer in the various solvents. Figures 3 and 4 compare the impact behaviors of the EGaIn droplets in water (polar solvent) and hexane (non-polar solvent) when they collide with the Si wafer. In water, the EGaIn droplet bounces off after collision while maintaining its spherical shape. The droplet in another polar solvent of DMSO also shows similar bouncing behavior (Figure S1 in Supplementary Materials). In ethanol, the EGaIn droplet does not show significant bouncing behavior but slightly adheres to the wafer (Figure S1). The slight adhesion prevents the EGaIn droplet from sliding down on the Si wafer tilted by up to $\sim 20^{\circ}$. (Figure S3). In contrast, in the non-polar solvents, hexane and benzene, the EGaIn droplets spread and adhere to the Si wafer upon collision (Figure 4 and Figure S2 in Supplementary Materials). In silicone oil, the EGaIn droplet neither bounces off nor adheres to the wafer. It gently lands on the wafer with high contact angle (Figure S2). The different adhesion behaviors of the EGaIn droplet in the non-polar solvents are observed when the wafer is tilted. In hexane and benzene, the EGaIn droplets adhere to the wafer and do not slide down even when the wafer is tilted by $22 \pm 1^{\circ}$, whereas the droplet rolls off in silicone oil when the wafer is tilted only by $10^{\circ}$ (Figure S3 in Supplementary Materials). 
(a)
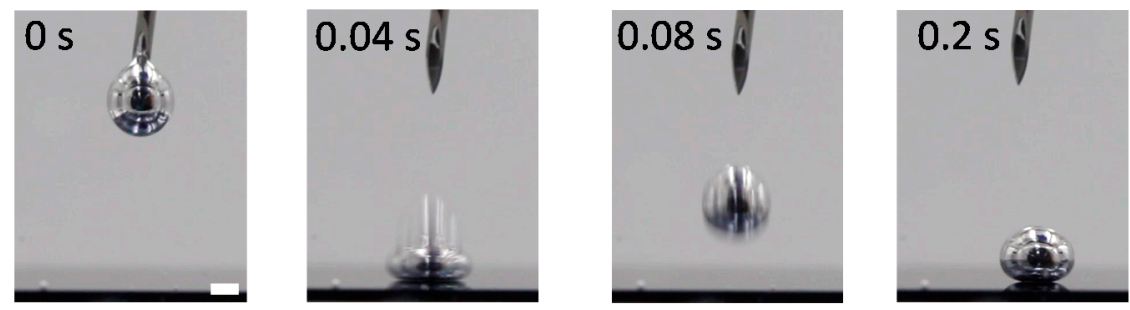

(b)
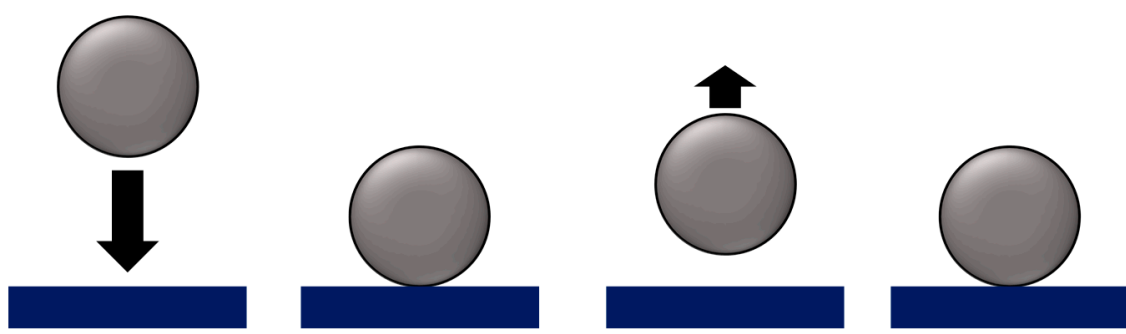

Figure 3. (a) Video snapshots of the EGaIn droplet impacting a Si wafer in water at $20^{\circ} \mathrm{C}$. Scale bar is $2 \mathrm{~mm}$. (b) Schematic of the "bouncing" behavior of the EGaIn droplet in (a).

(a)
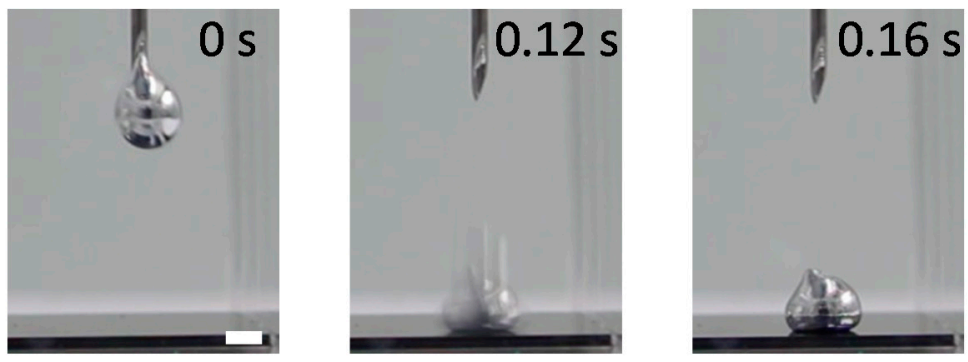

(b)

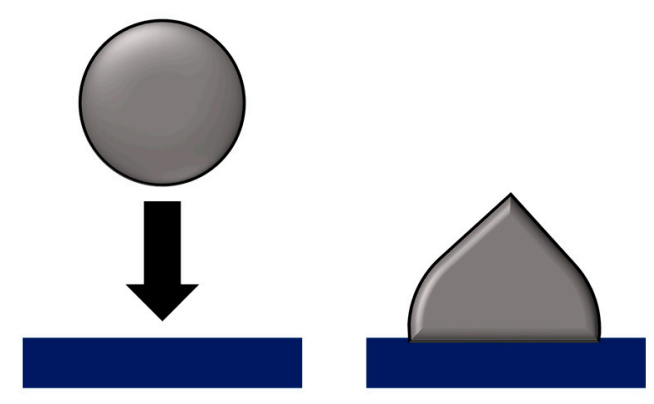

Figure 4. (a) Video snapshots of the EGaIn droplet impacting a Si wafer in hexane at $20{ }^{\circ} \mathrm{C}$. Scale bar is $2 \mathrm{~mm}$. (b) Schematic of the "adhesion" behavior of the EGaIn droplet in (a).

To explain the different impact dynamics of the EGaIn droplets depending on the solvent types, the Weber $(\mathrm{We}$ ) number can be utilized. The We number represents the ratio of the kinetic energy on impact to the interfacial energy and is defined by the following equation:

$$
W e=\frac{\rho v^{2} R_{0}}{\sigma},
$$

where $\rho$ is the density of the solvent, $v$ is the velocity on impact, $R_{0}$ is the droplet radius, and $\sigma$ is the interfacial tension of the droplet. Based on the We number, the impact behavior can be predicted; when the We number is larger, the impacting liquid droplet is more likely to spread to the substrate [34,35]. In our impact experiment, the interfacial tension between the EGaIn droplet and the surrounding solvent is the most crucial parameter to determine the We number and therefore the impact dynamics of the EGaIn droplet as other parameters do not change significantly. Because the We number is inversely proportional 
to the interfacial tension, the bouncing behavior of the EGaIn droplet in water and DMSO, i.e., low We number, means relatively high interfacial tension of the liquid metal in the polar solvents. In contrast, the adhesion behavior of the EGaIn in hexane and benzene, i.e., high We number, indicates low interfacial tension of EGaIn in the non-polar solvents. In the additional impact experiment in the air (Figure S4 in Supplementary Materials), the EGaIn droplet spreads out and sticks to the $\mathrm{Si}$ wafer similarly as in hexane or benzene. It is well known that the surface tension of EGaIn in the air dramatically decreases due to the formation of the gallium oxide surface layer [1-4]. In ethanol, the EGaIn droplet slightly adheres to the Si wafer without the bouncing behavior. This may be because the interfacial tension of EGaIn in ethanol is not as high as that in other polar solvents. It has been reported that EGaIn in ethanol is coated by a carbon layer that could act as a surfactant to reduce the interfacial tension of EGaIn [17]. In silicone oil, the EGaIn does not spread to the Si wafer but maintains a high contact angle. This should be mainly because the impact velocity is low in the highly viscous oil medium (viscosity $=100 \mathrm{mPa} \cdot \mathrm{s}$ ), resulting in a decrease in the We number. Thus, the interfacial energy between EGaIn and various solvents can be examined by analyzing the impact dynamics of the EGaIn droplets in the solvents.

Table 1 compares the molecular structures and properties of the six polar and nonpolar solvents and summarizes the experimental results of the interfacial characteristics of the EGaIn droplets in the solvents. The polar solvents, except for ethanol, have high surface tension, where the EGaIn droplet shows bouncing behavior on the Si wafer. The cohesive force between the solvent molecules is strong enough to prevent the droplet from adhering to the substrate and repel the droplet from the substrate. The ethanol has relatively low surface tension and presumably forms an interfacial layer, leading to the slight adhesion of EGaIn without any bouncing. The non-polar solvents tend to have low surface tension, where the EGaIn droplet spreads and adheres to the Si wafer. The silicone oil has extremely high viscosity compared to other solvents, which may prevent EGaIn from spreading and adhering to the wafer substrate. The interfacial behaviors of EGaIn can be correlated to Kamlet-Taft parameters, which include the information of basicity $(\beta)$ and polarity $\left(\pi^{*}\right)$ of solvents (Table 1) [36]. Figure 5 shows the correlation between interfacial behavior of the EGaIn droplet and the Kamlet-Taft parameters. As $\beta$ and $\pi^{*}$ increase, the EGaIn droplet tends to bounce off, whereas as $\beta$ and $\pi^{*}$ decrease, it is more likely to show strong adhesion to the substrate. Thus, the interfacial characteristics of EGaIn strongly depend on the types and surface tension values of surrounding solvents.

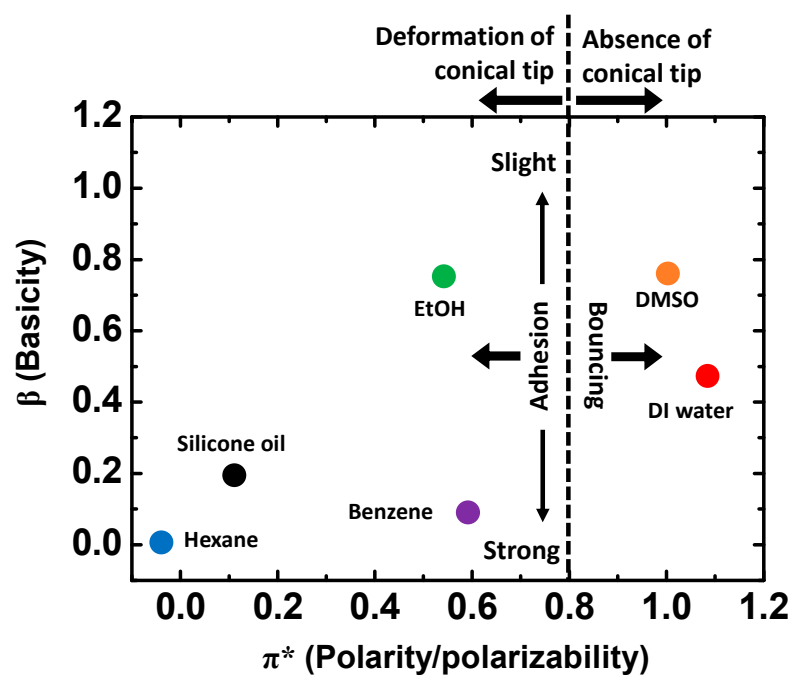

Figure 5. Interfacial behaviors depending on Kamlet-Taft parameters $\beta$ and $\pi^{*}$. 
Table 1. Molecular structures and physical properties of solvents used and interfacial characteristics of EGaIn in the corresponding solvents.

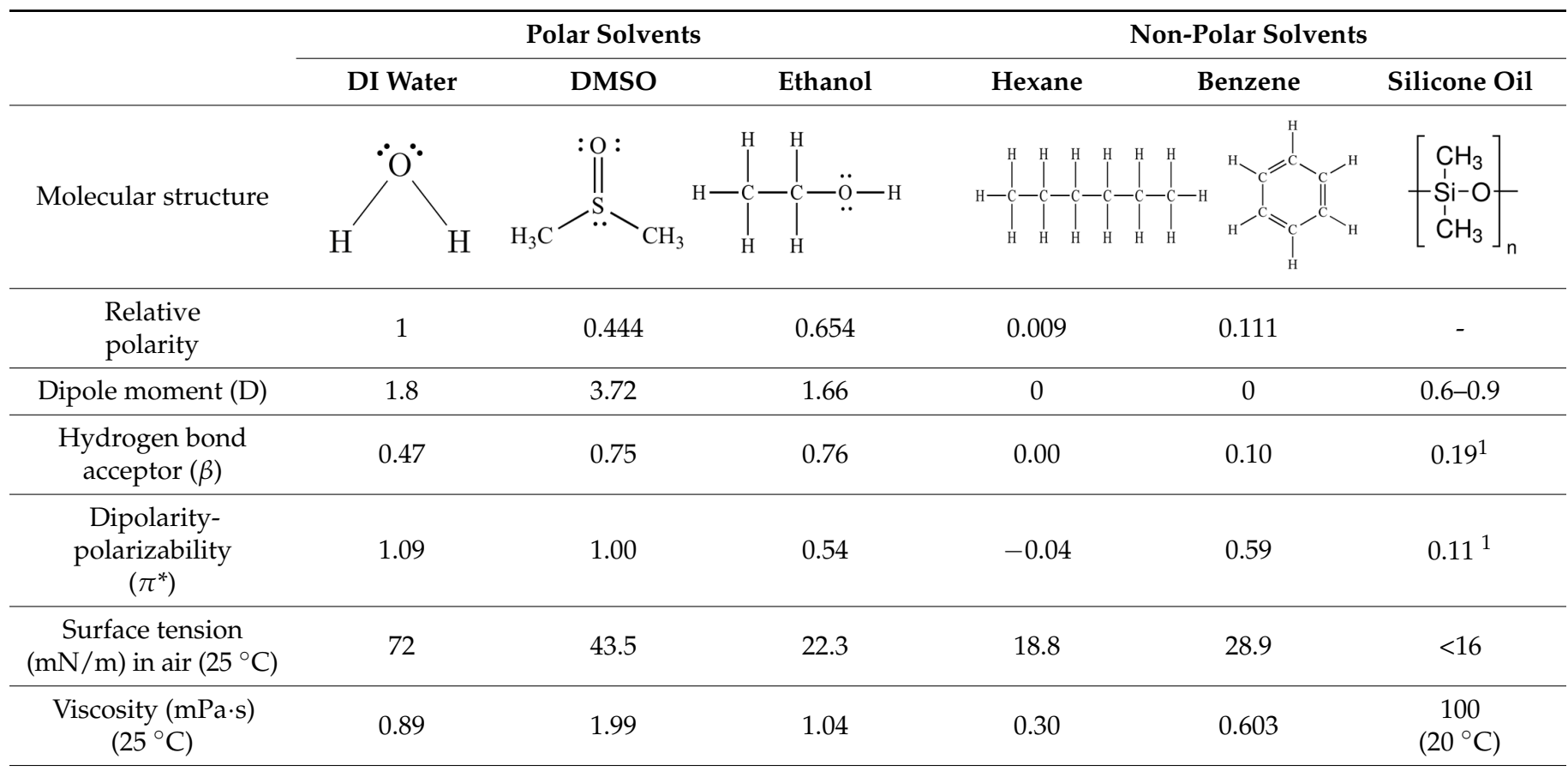

\begin{tabular}{|c|c|c|c|c|c|c|}
\hline \multicolumn{7}{|c|}{ Interfacial characteristics of the EGaIn droplets in corresponding solvents } \\
\hline $\begin{array}{c}\text { Contact angle on } \mathrm{Si} \\
\text { wafer }\left(^{\circ}\right)\end{array}$ & 158 & 160 & 158 & 158 & 159 & 160 \\
\hline Impact behavior & Bouncing & Bouncing & $\begin{array}{c}\text { Slight } \\
\text { adhesion }\end{array}$ & Adhesion & Adhesion & Sitting ${ }^{2}$ \\
\hline $\begin{array}{l}\text { Formation of conical } \\
\text { tip after dropping }\end{array}$ & $\times$ & $\times$ & $\triangle$ & $\mathrm{O}$ & $\mathrm{O}$ & $\mathrm{O}$ \\
\hline
\end{tabular}

In an aqueous environment, $\mathrm{pH}$ values could also be critical in determining the interfacial properties of EGaIn because, in strong acids or bases, the gallium-rich surface layer (e.g., gallium oxide and gallium oxide hydroxide) on EGaIn is susceptible to dissolution. We observed the EGaIn droplet in different $\mathrm{pH}$ solutions to understand how the $\mathrm{pH}$ value affects the interfacial properties of the EGaIn liquid metal in the aqueous environment. Figure 6a-c shows the contact angles of EGaIn droplets in $\mathrm{pH} 4,7$, and 10 buffer solutions. The droplets in all of the $\mathrm{pH}$ buffer solutions have high contact angles of $\sim 160^{\circ}$, which is comparable to the values previously discussed in Figure 1a. The shapes of the droplets vary depending on the $\mathrm{pH}$ of the buffer solutions. In $\mathrm{pH} 4$ and 7 buffers, the EGaIn droplets form the conical tips on top, whereas in $\mathrm{pH} 10$ buffer solution, the EGaIn droplet forms a spherical shape. This is because the surface layer of EGaIn droplet is dissolved in the strong basic condition of $\mathrm{pH} \mathrm{10,} \mathrm{whereas} \mathrm{the} \mathrm{acidity} \mathrm{of} \mathrm{the} \mathrm{pH} 4$ buffer solution is not strong enough to remove the surface layer thoroughly. As per the Pourbaix diagram, the gallium oxide skin of EGaIn is reduced at $\mathrm{pH}<3$ or $\mathrm{pH}>10[15,16]$. Interestingly, the EGaIn droplet in the $\mathrm{pH} 7$ buffer solution has the conical tip, which differs from that of the tip-less spherical EGaIn droplet in DI water (Figure 1b), although the DI water should also have a near-neutral $\mathrm{pH}$. We speculate that this may be due to the ions dissolved in the buffer solutions. A large amount of potassium, sodium, or phosphate ions might lower the interfacial energy between EGaIn and the aqueous buffer solution. 
(a)

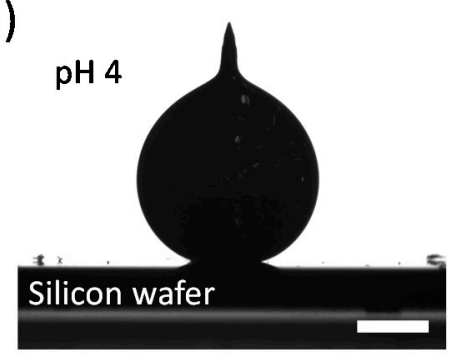

(b)

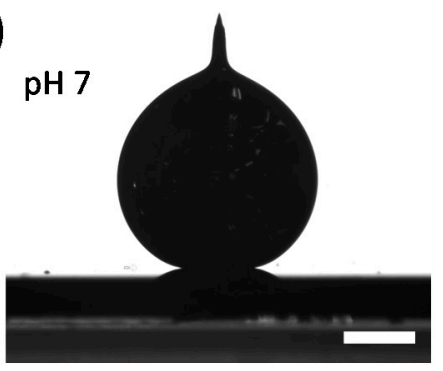

(c)

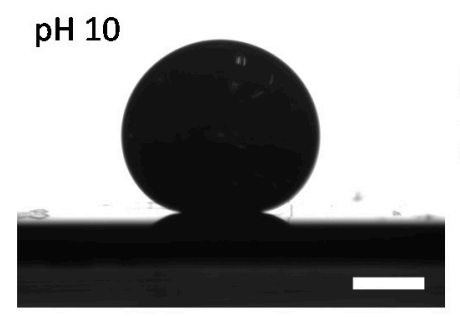

(d)

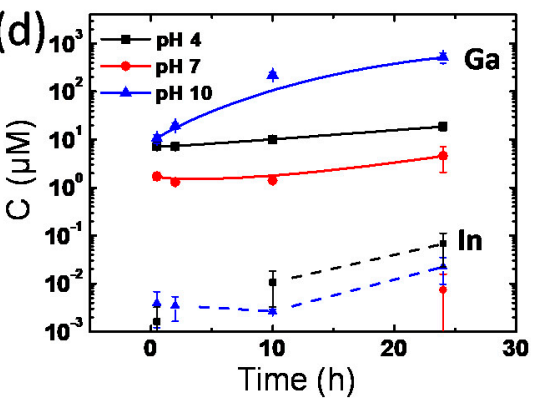

Figure 6. Side-view images of EGaIn droplets placed on a Si wafer in (a) $\mathrm{pH}$ 4, (b) $\mathrm{pH}$ 7. and (c) $\mathrm{pH}$ 10 buffer solutions at $20^{\circ} \mathrm{C}$. Scale bars are $0.5 \mathrm{~mm}$. (d) Time-dependent $\mathrm{Ga}$ and In ion concentrations dissolved into buffer solutions from the EGaIn liquid metal droplet.

To confirm the dissolution of the surface layer by the reduction in the $\mathrm{pH} 10$ buffer solution, the amount of $\mathrm{Ga}$ and In ions in each $\mathrm{pH}$ buffer solution in Figure 6a-c was measured and compared using mass spectroscopy. The time-dependent ion concentrations of $\mathrm{Ga}$ and In dissolved from EGaIn droplets in each $\mathrm{pH}$ buffer solution are shown in Figure $6 \mathrm{~d}$. In all $\mathrm{pH}$ conditions, $\mathrm{Ga}$ ions are more abundantly released from EGaIn than In ions, indicating that the surface layer is gallium-rich. Both concentrations of $\mathrm{Ga}$ and In ions increase with time but differ with $\mathrm{pH}$. The elution of $\mathrm{Ga}$ ions from EGaIn occurs most at $\mathrm{pH} 10$ and the least at $\mathrm{pH}$ 7. In the $\mathrm{pH} 10$ buffer solution, Ga ions eluted are up to $688 \mu \mathrm{M}$ in $24 \mathrm{~h}$, which is 112 times higher than the concentration of Ga ions eluted in the pH 7 solution. It is caused by the basic solution reducing gallium oxide to Ga ions. Thus, in an aqueous environment, the $\mathrm{pH}$ value is another crucial factor to determine the interfacial energy of EGaIn because of the $\mathrm{pH}$-dependent elution of the gallium-rich surface layer.

\section{Conclusions}

To summarize, we investigated the interfacial characteristics of EGaIn droplets in different types of polar and non-polar solvents. The difference in contact angles of the EGaIn droplets was insignificant, and the conical tip on top of the EGaIn droplets was formed in solvents with low surface tension. The impact dynamics of the EGaIn droplets in various solvents were analyzed where the droplet showed the different impact behaviors of bouncing, sitting, and adhesion after collision with the Si wafer. With the dimensionless We number, the different impact behaviors could be explained by the interfacial tension values of the EGaIn droplets in each solvent. In general, EGaIn tends to bounce off in polar solvents such as water and DMSO, representing high interfacial energy. In contrast, EGaIn spreads and adheres to the substrate in the non-polar solvents, indicating low interfacial energy. Furthermore, to investigate the $\mathrm{pH}$ effect on the interfacial behavior of EGaIn, the contact angles and shapes of the EGaIn droplets in $\mathrm{pH} \mathrm{4,} \mathrm{7,} \mathrm{and} 10$ buffer solutions were compared. The EGaIn droplet maintained the conical tip on top in $\mathrm{pH} 4$ and 7, whereas the metal droplet had a spherical shape in $\mathrm{pH} 10$ solution. This is presumably due to the $\mathrm{pH}$-dependent dissolution of the surface layer, which was supported by the measurement of the ions released from EGaIn in the buffer solutions. This research provides a useful guideline for selecting suitable solvents for using GaLMs depending on their fabrication processes and applications. Further study is now underway to investigate the chemical 
compositions and molecular structures of the surface layers formed on EGaIn surface in various solvents.

Supplementary Materials: The following supporting information can be downloaded at: https: / / www.mdpi.com/article/10.3390/ma15030706/s1, Figure S1: Video snapshots of a EGaIn droplet impacting on a Si wafer (a) in DMSO and (b) in ethanol. Scale bars are $2 \mathrm{~mm}$. The red arrows indicate the movement of EGaIn droplets; Figure S2: Video snapshots of a EGaIn droplet impacting on a Si wafer in (a) benzene and (b) silicone oil. Scale bars are $2 \mathrm{~mm}$; Figure S3: Video snapshots of EGaIn droplets when the wafer is tilted in (a) ethanol, (b) benzene, (c) hexane, and (d) silicone oil. Scale bars are $2 \mathrm{~mm}$. The tilting angles are $22 \pm 1^{\circ}$ in $(\mathrm{a}-\mathrm{c})$ and $10^{\circ}$ in (d); Figure S4. Video snapshots of a EGaIn droplet impacting on a Si wafer in the air. Scale bar is $2 \mathrm{~mm}$.

Author Contributions: Conceptualization, J.-H.S. and H.-J.K.; Funding acquisition, H.-J.K.; Investigation, J.-H.K., Y.-J.P. and S.K.; Supervision, J.-H.S. and H.-J.K.; Writing-original draft, J.-H.K., Y.-J.P., J.-H.S. and H.-J.K.; Writing-review \& editing, J.-H.S. and H.-J.K. All authors have read and agreed to the published version of the manuscript.

Funding: This study was supported by the Research Program funded by the SeoulTech (Seoul National University of Science and Technology).

Institutional Review Board Statement: Not applicable.

Informed Consent Statement: Not applicable.

Data Availability Statement: Not applicable.

Conflicts of Interest: The authors declare no conflict of interest.

\section{References}

1. Farrell, Z.J.; Tabor, C. Control of Gallium Oxide Growth on Liquid Metal Eutectic Gallium/Indium Nanoparticles via Thiolation. Langmuir 2018, 34, 234-240. [CrossRef]

2. Ren, L.; Zhuang, J.; Casillas, G.; Feng, H.; Liu, Y.; Xu, X.; Liu, Y.; Chen, J.; Du, Y.; Jiang, L.; et al. Nanodroplets for Stretchable Superconducting Circuits. Adv. Funct. Mater. 2016, 26, 8111-8118. [CrossRef]

3. Song, H.; Kim, T.; Kang, S.; Jin, H.; Lee, K.; Yoon, H.J. Ga-Based Liquid Metal Micro/Nanoparticles: Recent Advances and Applications. Small 2020, 16, 1903391. [CrossRef] [PubMed]

4. Wang, P.-F.; Hu, Q.; Lv, B.; Zhu, J.-L.; Ma, W.; Dong, Z.; Wei, J.; Sun, J.-L. Facile fabrication of eutectic gallium-indium alloy nanostructure and application in photodetection. Nanotechnology 2020, 31, 145703. [CrossRef]

5. Shu, J.; Lu, Y.; Wang, E.; Li, X.; Tang, S.-Y.; Zhao, S.; Zhou, X.; Sun, L.; Li, W.; Zhang, S. Particle-Based Porous Materials for the Rapid and Spontaneous Diffusion of Liquid Metals. ACS Appl. Mater. Interfaces 2020, 12, 11163-11170. [CrossRef]

6. $\quad$ Lu, Y.; Hu, Q.; Lin, Y.; Pacardo, D.B.; Wang, C.; Sun, W.; Ligler, F.S.; Dickey, M.; Gu, Z. Transformable liquid-metal nanomedicine. Nat. Commun. 2015, 6, 10066. [CrossRef]

7. Zhu, S.; So, J.-H.; Mays, R.; Desai, S.; Barnes, W.R.; Pourdeyhimi, B.; Dickey, M.D. Ultrastretchable Fibers with Metallic Conductivity Using a Liquid Metal Alloy Core. Adv. Funct. Mater. 2013, 23, 2308-2314. [CrossRef]

8. Do, T.N.; Visell, Y. Stretchable, Twisted Conductive Microtubules for Wearable Computing, Robotics, Electronics, and Healthcare. Sci. Rep. 2017, 7, 1753. [CrossRef]

9. Dickey, D.M. Stretchable and Soft Electronics using Liuqid Metals. Adv. Mater. 2017, 29, 1606425. [CrossRef] [PubMed]

10. Daeneke, T.; Khoshmanesh, K.; Mahmood, N.; de Castro, I.A.; Esrafilzadeh, D.; Barrow, S.J.; Dickey, M.D.; Kalantar-Zadeh, K. Liquid metals: Fundamentals and applications in chemistry. Chem. Soc. Rev. 2018, 47, 4073-4111. [CrossRef] [PubMed]

11. Holcomb, S.; Brothers, M.; Diebold, A.; Thatcher, W.; Mast, D.; Tabor, C.; Heikenfeld, J. Oxide-Free Actuation of Gallium Liquid Metal Alloys Enabled by Novel Acidified Siloxane Oils. Langmuir 2016, 32, 12656-12663. [CrossRef] [PubMed]

12. Hutter, T.; Bauer, W.-A.C.; Elliott, S.R.; Huck, W.T.S. Formation of Spherical and Non-Spherical Eutectic Gallium-Indium Liquid-Metal Microdroplets in Microfluidic Channels at Room Temperature. Adv. Funct. Mater. 2012, 22, 2624-2631. [CrossRef]

13. Lin, Y.; Liu, Y.; Genzer, J.; Dickey, M.D. Shape-transformable liquid metal nanoparticles in aqueous solution. Chem. Sci. 2017, 8, 3832-3837. [CrossRef] [PubMed]

14. Horasawa, N.; Nakajima, H.; Takahashi, S.; Okabe, T. Behavior of Pure Gallium in Water and Various Saline Solutions. Dent. Mater. J. 1997, 16, 200-208. [CrossRef] [PubMed]

15. Pourbaix, M. Atlas of electrochemical equilibria in aqueous solution. NACE 1974, 307, 428-448.

16. Dickey, M.D. Emerging Applications of Liquid Metals Featuring Surface Oxides. ACS Appl. Mater. Interfaces 2014, 6, 18369-18379. [CrossRef] [PubMed]

17. Lin, Y.; Cooper, C.; Wang, M.; Adams, J.J.; Genzer, J.; Dickey, M.D. Handwritten, Soft Circuit Boards and Antennas Using Liquid Metal Nanoparticles. Small 2015, 11, 6397-6403. [CrossRef] 
18. Zhang, C.; McKeon, L.; Kremer, M.P.; Park, S.-H.; Ronan, O.; Seral-Ascaso, A.; Barwich, S.; Coileáin, C.Ó.; McEvoy, N.; Nerl, H.C.; et al. Additive-free MXene inks and direct printing of micro-supercapacitors. Nat. Commun. 2019, 10, 1795. [CrossRef]

19. Zhang, M.; Li, G.; Huang, L.; Ran, P.; Huang, J.; Yu, M.; Yuqian, H.; Guo, J.; Liu, Z.; Ma, X. Versatile fabrication of liquid metal nano-ink based flexible electronic devices. Appl. Mater. Today 2021, 22, 100903. [CrossRef]

20. Neumann, T.V.; Facchine, E.G.; Leonardo, B.; Khan, S.; Dickey, M.D. Direct write printing of a self-encapsulating liquid metalsilicone composite. Soft Matter 2020, 16, 6608-6618. [CrossRef]

21. Reichardt, C.; Welton, T. Solvent effects on the absorption spectra of organic compounds. In Solvents and Solvent Effects in Organic Chemistry, 1st ed.; Wiley-VCH Verlag: Weinheim, Germany, 2011; pp. 359-424. [CrossRef]

22. Zhou, F.; Shao, J.; Yang, Y.; Zhao, J.; Guo, H.; Li, X.; Ji, S.; Zhang, Z. Molecular Rotors as Fluorescent Viscosity Sensors: Molecular Design, Polarity Sensitivity, Dipole Moments Changes, Screening Solvents, and Deactivation Channel of the Excited States. Eur. J. Org. Chem. 2011, 2011, 4773-4787. [CrossRef]

23. Lee, J.N.; Park, A.C.; Whitesides, G.M. Solvent Compatibility of Poly(dimethylsiloxane)-Based Microfluidic Devices. Anal. Chem. 2003, 75, 6544-6554. [CrossRef] [PubMed]

24. Bonfante, G.; Chevalliot, S.; Toury, B.; Berge, B.; Maillard, M. Two-liquid wetting properties as a surface polarity probe for hydrophobic coatings. Phys. Chem. Chem. Phys. 2017, 19, 3214-3218. [CrossRef] [PubMed]

25. Chen, G.; Wang, H.; Guo, R.; Duan, M.; Zhang, Y.; Liu, J. Superelastic EGaIn Composite Fibers Sustaining 500\% Tensile Strain with Superior Electrical Conductivity for Wearable Electronics. ACS Appl. Mater. Interfaces 2020, 12, 6112-6118. [CrossRef]

26. Guo, R.; Tang, J.; Dong, S.; Lin, J.; Wang, H.; Liu, J.; Rao, W. One-Step Liquid Metal Transfer Printing: Toward Fabrication of Flexible Electronics on Wide Range of Substrates. Adv. Mater. Technol. 2018, 3, 1800265. [CrossRef]

27. Li, H.; Qiao, R.; Davis, T.; Tang, S.-Y. Biomedical Applications of Liquid Metal Nanoparticles: A Critical Review. Biosensors 2020, 10, 196. [CrossRef] [PubMed]

28. Joshipura, I.D.; Persson, K.A.; Truong, V.K.; Oh, J.-H.; Kong, M.; Vong, M.H.; Ni, C.; Alsafatwi, M.; Parekh, D.P.; Zhao, H.; et al. Are Contact Angle Measurements Useful for Oxide-Coated Liquid Metals? Langmuir 2021, 37, 10914-10923. [CrossRef] [PubMed]

29. Ikuno, T.; Somei, Z. Fabrication of Eutectic Ga-In Nanowire Arrays Based on Plateau-Rayleigh Instability. Molecules 2021, $26,4616$. [CrossRef]

30. Tang, S.; Qiao, R.; Lin, Y.; Li, Y.; Zhao, Q.; Yuan, D.; Yun, G.; Guo, J.; Dickey, M.; Huang, T.J.; et al. Functional Liquid Metal Nanoparticles Produced by Liquid-Based Nebulization. Adv. Mater. Technol. 2019, 4, 1800420. [CrossRef]

31. Bowers, C.M.; Liao, K.-C.; Yoon, H.J.; Rappoport, D.; Baghbanzadeh, M.; Simeone, F.C.; Whitesides, G.M. Introducing Ionic and/or Hydrogen Bonds into the SAM/ / $\mathrm{Ga}_{2} \mathrm{O}_{3}$ Top-Interface of $\mathrm{Ag}^{\mathrm{TS}} / \mathrm{S}\left(\mathrm{CH}_{2}\right)_{\mathrm{n}} \mathrm{T} / / \mathrm{Ga}_{2} \mathrm{O}_{3} / \mathrm{EGaIn}$ Junctions. Nano Lett. 2014, 14, 3521-3526. [CrossRef]

32. Chen, L.; Wang, Y.; Peng, X.; Zhu, Q.; Zhang, K. Impact Dynamics of Aqueous Polymer Droplets on Superhydrophobic Surfaces Macromolecules 2018, 51, 7817-7827. [CrossRef]

33. Antonini, C.; Villa, F.; Marengo, M. Oblique impacts of water drops onto hydrophobic and superhydrophobic surfaces: Outcomes, timing, and rebound maps. Exp. Fluids 2014, 55, 1713. [CrossRef]

34. Baek, S.; Moon, H.S.; Kim, W.; Jeon, S.; Yong, K. Effect of liquid droplet surface tension on impact dynamics over hierarchical nanostructure surfaces. Nanoscale 2018, 10, 17842-17851. [CrossRef] [PubMed]

35. Clanet, C.; Béguin, C.; Richard, D.; Quéré, D. Maximal deformation of an impacting drop. J. Fluid Mech. 2004, 517, 199-208. [CrossRef]

36. Komorova, A.O.; Dick, G.R.; Luterbacher, J.S. Diformylxylose as a new polar aprotic solvent produced form renweable biomass. Green Chem. 2021, 23, 4790. [CrossRef] 